\title{
WATER PRODUCTION IN COMETS 2001 Q4 (NEAT) AND 2002 T7 (LINEAR) DETERMINED FROM SOHO/SWAN OBSERVATIONS
}

\author{
M. R. Combi ${ }^{1}$, J. T. T. MäKinen ${ }^{2}$, J.-L. Bertaux $^{3}$, Y. LeE ${ }^{1}$, And E. Quémerais ${ }^{3}$ \\ ${ }^{1}$ Department of Atmospheric, Oceanic and Space Sciences, University of Michigan, 2455 Hayward Street, Ann Arbor, MI 48109-2143, USA; mcombi@ umich.edu \\ ${ }^{2}$ Finnish Meteorological Institute, Box 503, SF-00101 Helsinki, Finland \\ ${ }^{3}$ Centre National de la Recherche Scientifique, Service d'Aéronomie, BP3, 91371 Verrières le Buisson Cedex, France \\ Received 2008 November 25; accepted 2009 March 9; published 2009 April 27
}

\begin{abstract}
S
The SWAN all-sky camera on the Solar and Heliospheric Observatory (SOHO) spacecraft detected the hydrogen Lyman-alpha $(\mathrm{Ly} \alpha)$ comae of comets 2001 Q4 NEAT and 2002 T7 LINEAR for large portions of their perihelion apparitions in 2003 and 2004. C/2001 Q4 NEAT was observed from 2003 September 14 through 2004 November 2 , covering heliocentric distances from 3.23 AU before perihelion to 2.75 AU after, and C/2002 T7 LINEAR was observed from 2003 December 4 through 2004 August 6, covering heliocentric distances from 2.52 AU before perihelion to $2.09 \mathrm{AU}$ after. We combined the full set of comet specific and full-sky observations and used our time-resolved model (TRM), which enables us to extract continuous values of the daily-average value of the water production rate throughout most of this entire period. The average power-law fit to the production rate variation of C/2001 Q4 NEAT with heliocentric distance, $r$, gives $3.5 \times 10^{29} r^{-1.7}$ and that for C/2002 T7 LINEAR gives $4.6 \times$ $10^{29} r^{-2.0}$. Both comets show roughly a factor of 2 asymmetry in activity about perihelion, being more active before perihelion. C/2001 Q4 NEAT showed a production rate outburst about 30 days before perihelion (2004 April 15) and then a large extended increase above the nominal trend from 50 to 70 days after perihelion (2004 July 5-July 25).
\end{abstract}

Key words: comets: general - comets: individual (C/2001 Q4 (NEAT), C/2002 T7 (LINEAR)) - Oort Cloud

\section{INTRODUCTION}

Being stationed at the L1 Lagrange point 1.5 million $\mathrm{km}$ in front of the Earth, the Solar and Heliospheric Observatory $(\mathrm{SOHO})$ spacecraft has been well positioned for 13 years to obtain continuous temporal coverage of the Sun and solar wind. The SOHO Solar Wind Anisotropies (SWAN) camera, makes all-sky Lyman-alpha $(\operatorname{Ly} \alpha)$ images of the hydrogen distribution in the interplanetary medium (IPM), typically in the brightness range of 500-1000 Rayleighs, providing a global picture of the solar wind, which shapes the hydrogen distribution by charge impact ionization (Bertaux et al. 1997).

Because solar ultraviolet radiation dissociates cometary water and its byproduct $\mathrm{OH}$, producing two hydrogen atoms per molecule, comets are surrounded by a large atomic hydrogen coma, which being illuminated by solar Ly $\alpha$ photons at $1215.7 \AA$, presents a useful target for monitoring the water production rate in comets. Obtaining accurate water production rates in comets is particularly important because water is usually the most abundant parent gas species in the coma, controlling the overall activity, because measurements of all other species are compared to it for compositional interpretation, and because of the crucial role of comets in the larger issue of the origin of the solar system. Furthermore, we have developed a method of simultaneously analyzing sets of SWAN images, usually recorded a few times per week, to extract daily average values of the water production rate by the nucleus (Mäkinen \& Combi 2005). Using the extracted daily-average values we have compared with various complementary data sets (Combi et al. 2005, 2008) and found reasonable agreement with both long-term and short-term variations of the cometary water production, including large outbursts.

This analysis procedure combines and extends the best and necessary parts of the syndyname approaches of Keller and Meier (1976), the vectorial model of Festou (1981), and a parameterized version of the collisional physics Monte Carlo models of Combi and Smyth (1988) into a reasonably computationally fast tool for analyzing both single SWAN images as well as a series of the wide-field observations of the H Ly $\alpha$ coma. The model builds up time-tagged, sky-plane basis functions of $\mathrm{H}$ Ly $\alpha$ for the times and various locations within a whole set of SWAN images that correspond to a certain production rate of water molecules from the nucleus. By employing a standard inversion technique (the Singular Value Decomposition), the time-resolved model (TRM) can be used to calculate either an average production rate for a single image or to deconvolve a whole set of images into water production rates at some desired time-resolution between many images (Mäkinen \& Combi 2005). Given the field of view of SWAN and photochemical lifetimes of water and $\mathrm{OH}$, usually daily-average values of the water production rate at the nucleus can be extracted from a series of images taken at a rate of 2-4 per week. Using this procedure we were able to analyze a large set of Ly $\alpha$ images of comet 1996 B2 Hyakutake and resolve three major outbursts including the well-known 1996 March 19 outburst. We have since used the TRM for several other comets for both single-image production rates (Mäkinen et al. 2007; Neugebauer et al. 2007) and daily averages (Combi et al. 2008).

Here, we present the results of a TRM analysis of more than a year of SWAN observations in 2003 and 2004 of comets C/2001 Q4 (NEAT), hereafter referred to as Q4, and eight months of observations of $\mathrm{C} / 2002 \mathrm{~T} 7$ (LINEAR), hereafter referred to as T7. These comets were discovered by two of the near Earth object (NEO) survey telescopes while they were quite far from the Sun in 2001 and 2002, and both were anticipated and well planned observationally. Comet 2001 Q4 NEAT was observed by SWAN from 2003 September 14 through 2004 November 2, covering heliocentric distances from 3.23 AU before perihelion to $2.75 \mathrm{AU}$ after. Comet $2002 \mathrm{~T} 7 \mathrm{LINEAR}$ was observed from 2003 December 4 through 2004 August 6, covering heliocentric distances from 2.52 AU before perihelion to 2.09 AU after. With the TRM we calculated both single-image 
Table 1

C/2002 T7 LINEAR: Observational Circumstances and Single-Image Water Production Rates

\begin{tabular}{|c|c|c|c|c|c|}
\hline$\Delta T$ (days) & $r(\mathrm{AU})$ & $\Delta(\mathrm{AU})$ & $g\left(\mathrm{~s}^{-1}\right)$ & $Q\left(10^{28} \mathrm{~s}^{-1}\right)$ & $\delta Q\left(10^{28} \mathrm{~s}^{-1}\right)$ \\
\hline-141.379 & 2.515 & 1.602 & 0.001716 & 8.981 & 0.02 \\
\hline-140.323 & 2.501 & 1.596 & 0.001715 & 4.206 & 0.05 \\
\hline-139.268 & 2.487 & 1.590 & 0.001714 & 13.17 & 0.01 \\
\hline-138.212 & 2.473 & 1.585 & 0.001714 & 6.722 & 0.03 \\
\hline-136.096 & 2.444 & 1.576 & 0.001713 & 15.72 & 0.01 \\
\hline-128.704 & 2.344 & 1.568 & 0.001709 & 15.97 & 0.01 \\
\hline-127.649 & 2.329 & 1.569 & 0.001708 & 23.83 & 0.01 \\
\hline-126.593 & 2.315 & 1.571 & 0.001707 & 10.16 & 0.02 \\
\hline-125.537 & 2.300 & 1.573 & 0.001707 & 18.62 & 0.01 \\
\hline-124.481 & 2.286 & 1.576 & 0.001706 & 14.64 & 0.01 \\
\hline-123.425 & 2.271 & 1.580 & 0.001706 & 15.02 & 0.01 \\
\hline-122.368 & 2.257 & 1.583 & 0.001705 & 16.55 & 0.007 \\
\hline-121.313 & 2.242 & 1.588 & 0.001705 & 20.46 & 0.007 \\
\hline-120.257 & 2.228 & 1.592 & 0.001725 & 16.33 & 0.006 \\
\hline-119.201 & 2.213 & 1.598 & 0.001724 & 16.82 & 0.01 \\
\hline-118.145 & 2.198 & 1.603 & 0.001724 & 15.75 & 0.007 \\
\hline-106.606 & 2.036 & 1.685 & 0.001718 & 21.43 & 0.008 \\
\hline-106.289 & 2.031 & 1.687 & 0.001718 & 18.98 & 0.002 \\
\hline-104.601 & 2.007 & 1.702 & 0.001717 & 23.05 & 0.008 \\
\hline-102.792 & 1.981 & 1.717 & 0.001716 & 25.80 & 0.006 \\
\hline-100.498 & 1.948 & 1.738 & 0.001715 & 32.26 & 0.007 \\
\hline-98.689 & 1.922 & 1.754 & 0.001734 & 30.57 & 0.006 \\
\hline-96.887 & 1.896 & 1.770 & 0.001733 & 27.77 & 0.007 \\
\hline-95.078 & 1.869 & 1.786 & 0.001733 & 28.16 & 0.007 \\
\hline-92.787 & 1.836 & 1.807 & 0.001731 & 25.45 & 0.008 \\
\hline-90.986 & 1.809 & 1.823 & 0.001730 & 28.79 & 0.008 \\
\hline-89.177 & 1.783 & 1.839 & 0.001730 & 27.83 & 0.007 \\
\hline-86.883 & 1.749 & 1.858 & 0.001728 & 29.59 & 0.009 \\
\hline-85.073 & 1.722 & 1.873 & 0.001727 & 28.81 & 0.009 \\
\hline-57.790 & 1.307 & 2.007 & 0.001736 & 54.74 & 0.007 \\
\hline-54.265 & 1.252 & 2.007 & 0.001735 & 82.21 & 0.001 \\
\hline-53.512 & 1.240 & 2.006 & 0.001734 & 58.37 & 0.007 \\
\hline-51.882 & 1.215 & 2.004 & 0.001733 & 47.94 & 0.002 \\
\hline-50.514 & 1.194 & 2.001 & 0.001733 & 54.89 & 0.007 \\
\hline-48.716 & 1.166 & 1.996 & 0.001732 & 56.29 & 0.007 \\
\hline-46.572 & 1.133 & 1.988 & 0.001731 & 60.62 & 0.007 \\
\hline-43.586 & 1.087 & 1.973 & 0.001730 & 56.36 & 0.008 \\
\hline-41.774 & 1.059 & 1.961 & 0.001729 & 72.35 & 0.009 \\
\hline-39.641 & 1.026 & 1.946 & 0.001728 & 83.77 & 0.007 \\
\hline-7.637 & 0.637 & 1.354 & 0.001472 & 174.6 & 0.002 \\
\hline-6.138 & 0.629 & 1.308 & 0.001464 & 118.0 & 0.009 \\
\hline-4.717 & 0.623 & 1.262 & 0.001450 & 128.7 & 0.001 \\
\hline-3.923 & 0.621 & 1.236 & 0.001444 & 119.7 & 0.007 \\
\hline 6.320 & 0.630 & 0.866 & 0.001422 & 74.91 & 0.004 \\
\hline 7.375 & 0.635 & 0.826 & 0.001425 & 93.47 & 0.003 \\
\hline 9.814 & 0.651 & 0.732 & 0.001443 & 96.70 & 0.003 \\
\hline 13.148 & 0.678 & 0.605 & 0.001468 & 66.05 & 0.003 \\
\hline 15.919 & 0.705 & 0.502 & 0.001486 & 69.78 & 0.003 \\
\hline 18.292 & 0.731 & 0.420 & 0.001506 & 55.07 & 0.003 \\
\hline 36.424 & 0.978 & 0.498 & 0.001577 & 23.83 & 0.004 \\
\hline 39.438 & 1.023 & 0.605 & 0.001591 & 26.77 & 0.005 \\
\hline 41.251 & 1.051 & 0.671 & 0.001590 & 23.67 & 0.005 \\
\hline 43.396 & 1.084 & 0.750 & 0.001589 & 22.44 & 0.007 \\
\hline 67.583 & 1.458 & 1.619 & 0.001582 & 9.331 & 0.02 \\
\hline 69.250 & 1.483 & 1.676 & 0.001582 & 10.40 & 0.02 \\
\hline 71.250 & 1.514 & 1.743 & 0.001581 & 6.999 & 0.03 \\
\hline 74.250 & 1.559 & 1.842 & 0.001581 & 8.441 & 0.03 \\
\hline 75.286 & 1.575 & 1.876 & 0.001580 & 9.533 & 0.03 \\
\hline 76.342 & 1.591 & 1.910 & 0.001580 & 11.46 & 0.03 \\
\hline 84.480 & 1.713 & 2.164 & 0.001564 & 13.18 & 0.03 \\
\hline 86.855 & 1.748 & 2.236 & 0.001563 & 5.425 & 0.06 \\
\hline 88.605 & 1.774 & 2.288 & 0.001563 & 11.17 & 0.03 \\
\hline 89.479 & 1.787 & 2.313 & 0.001563 & 7.181 & 0.008 \\
\hline 95.598 & 1.877 & 2.487 & 0.001561 & 8.706 & 0.009 \\
\hline 100.849 & 1.953 & 2.629 & 0.001546 & 8.373 & 0.06 \\
\hline
\end{tabular}

Table 1

(Continued)

\begin{tabular}{lccccc}
\hline \hline$\Delta T$ (days $)$ & $r(\mathrm{AU})$ & $\Delta(\mathrm{AU})$ & $g\left(\mathrm{~s}^{-1}\right)$ & $Q\left(10^{28} \mathrm{~s}^{-1}\right)$ & $\delta Q\left(10^{28} \mathrm{~s}^{-1}\right)$ \\
\hline 101.465 & 1.962 & 2.645 & 0.001546 & 5.376 & 0.01 \\
102.599 & 1.978 & 2.674 & 0.001546 & 9.510 & 0.04 \\
105.474 & 2.019 & 2.748 & 0.001545 & 12.67 & 0.05 \\
\hline
\end{tabular}

Notes.

$\Delta T$ : time from perihelion 2004 April 23.0623, in days.

$r$ : heliocentric distance (AU).

$\Delta$ : geocentric distance (AU).

$g$ : solar Ly $\alpha$-factor (photons s ${ }^{-1}$ ).

$Q$ : water production rate $\left(10^{28} \mathrm{~s}^{-1}\right)$.

$\delta Q: 1 \sigma$ uncertainty $\left(10^{28} \mathrm{~s}^{-1}\right)$.

production rates as well as deconvolved daily average values, covering the activity of Q4 and T7 for long continuous intervals of time. We examine the pre- and post-perihelion activity and the variation of activity with heliocentric distance. We also compare the water production rates with published results of water production rate from observations of water and its other photodissociation products. Finally, we discuss the variation with heliocentric distance with the expanding set of comets observed in this manner with SWAN.

\section{TRM ANALYSIS RESULTS}

Tables 1 and 2 give the observational circumstances, and Figures 1 and 2 show the entire set of single-image water production rates for $\mathrm{T} 7$ and $\mathrm{Q} 4$, respectively. The production rate values at the time the image was obtained are extracted assuming only the long-term heliocentric distance dependent variation of water production rate within the image. It is clear that both of these comets were just over a factor of 2 more productive at comparable heliocentric distances before perihelion compared to after. On the other hand, the overall slopes of the variations before and after perihelion are similar for each comet. The average power-law fit to the production rate variation of $\mathrm{Q} 4$ gives $3.5 \times 10^{29} r^{-1.7}$ and that for T7 gives $4.6 \times 10^{29} r^{-2.0}$. Please note the power-law fits do include all the data as given, including any short-term variations such as outbursts. One advantage of SWAN having both wide and continuous coverage is that we can to a certain extent average over short-term activity variations.

Tables 3 and 4 and Figures 3 and 4 give the TRM dailyaverage deconvolved water production rates for $\mathrm{T} 7$ and $\mathrm{Q} 4$, respectively. For both comets there are sporadic gaps in the daily-average coverage, normally when the comet is too close to bright stars, e.g., near the galactic equator. There are not many water production rate values for these comets available in the published literature yet, but those that are available are plotted with the SWAN results. The Odin microwave observations (Biver et al. 2007) agree quite well with the T7 results. Those for Q4 are somewhat below the SWAN results. Preliminary results from infrared observations of water (Anderson et al. 2008) are in excellent agreement for T7. Their one value for Q4 is almost a factor of two above the SWAN values. J. Crovisier et al. (2009, in preparation) have provided preliminary results of $\mathrm{OH} 18 \mathrm{~cm}$ observations of Q4 from Nançay. This range of differences in consistency is similar to those found between SWAN results and other methodologies (water in the IR, OH in near UV and radio, etc.) in the SWAN results for the five moderately bright comets from 1999 to 2001 (Combi et al. 2008). 
Table 2

C/2001 Q4 NEAT: Observational Circumstances and Single-Image Water Production Rates

\begin{tabular}{|c|c|c|c|c|c|}
\hline$\Delta T$ (days) & $r(\mathrm{AU})$ & $\overline{\Delta \Delta(\mathrm{AU})}$ & $g\left(\mathrm{~s}^{-1}\right)$ & $Q\left(10^{28} \mathrm{~s}^{-1}\right)$ & $\delta Q\left(10^{28} \mathrm{~s}^{-1}\right)$ \\
\hline-212.872 & 3.233 & 2.975 & 0.001674 & 1.525 & 0.13 \\
\hline-208.976 & 3.189 & 2.950 & 0.001672 & 1.850 & 0.022 \\
\hline-206.326 & 3.159 & 2.935 & 0.001671 & 2.236 & 0.089 \\
\hline-192.309 & 2.997 & 2.865 & 0.001678 & 3.480 & 0.054 \\
\hline-175.881 & 2.805 & 2.802 & 0.001669 & 4.135 & 0.067 \\
\hline-173.770 & 2.780 & 2.794 & 0.001668 & 4.585 & 0.052 \\
\hline-172.725 & 2.768 & 2.790 & 0.001667 & 2.847 & 0.080 \\
\hline-171.669 & 2.756 & 2.787 & 0.001666 & 6.978 & 0.036 \\
\hline-168.507 & 2.718 & 2.775 & 0.001665 & 9.365 & 0.024 \\
\hline-166.406 & 2.693 & 2.767 & 0.001664 & 7.121 & 0.036 \\
\hline-165.355 & 2.681 & 2.763 & 0.001663 & 4.932 & 0.045 \\
\hline-163.243 & 2.656 & 2.755 & 0.001662 & 6.446 & 0.035 \\
\hline-162.197 & 2.643 & 2.750 & 0.001662 & 3.790 & 0.059 \\
\hline-161.141 & 2.630 & 2.746 & 0.001661 & 4.699 & 0.050 \\
\hline-159.035 & 2.605 & 2.737 & 0.001660 & 2.550 & 0.089 \\
\hline-157.979 & 2.593 & 2.733 & 0.001659 & 3.078 & 0.081 \\
\hline-156.933 & 2.580 & 2.728 & 0.001658 & 4.907 & 0.053 \\
\hline-151.659 & 2.517 & 2.704 & 0.001656 & 8.876 & 0.027 \\
\hline-149.557 & 2.491 & 2.693 & 0.001655 & 8.606 & 0.029 \\
\hline-148.506 & 2.479 & 2.688 & 0.001654 & 5.903 & 0.039 \\
\hline-147.450 & 2.466 & 2.682 & 0.001654 & 7.778 & 0.030 \\
\hline-146.395 & 2.453 & 2.677 & 0.001653 & 11.17 & 0.024 \\
\hline-145.348 & 2.440 & 2.671 & 0.001653 & 8.664 & 0.030 \\
\hline-144.292 & 2.427 & 2.665 & 0.001669 & 6.619 & 0.033 \\
\hline-143.237 & 2.415 & 2.659 & 0.001669 & 10.03 & 0.027 \\
\hline-142.186 & 2.402 & 2.653 & 0.001668 & 9.701 & 0.026 \\
\hline-141.130 & 2.389 & 2.646 & 0.001668 & 6.309 & 0.042 \\
\hline-129.546 & 2.247 & 2.565 & 0.001662 & 9.312 & 0.017 \\
\hline-127.527 & 2.223 & 2.549 & 0.001661 & 12.73 & 0.013 \\
\hline-125.714 & 2.200 & 2.533 & 0.001660 & 14.02 & 0.013 \\
\hline-123.408 & 2.172 & 2.513 & 0.001659 & 12.64 & 0.015 \\
\hline-121.596 & 2.150 & 2.496 & 0.001658 & 16.90 & 0.011 \\
\hline-119.783 & 2.127 & 2.479 & 0.001657 & 18.47 & 0.0097 \\
\hline-117.970 & 2.105 & 2.461 & 0.001656 & 16.59 & 0.011 \\
\hline-115.665 & 2.076 & 2.437 & 0.001655 & 18.64 & 0.0090 \\
\hline-113.852 & 2.054 & 2.418 & 0.001654 & 18.93 & 0.0086 \\
\hline-112.039 & 2.031 & 2.397 & 0.001653 & 18.28 & 0.0088 \\
\hline-109.734 & 2.003 & 2.371 & 0.001652 & 18.48 & 0.0085 \\
\hline-107.921 & 1.980 & 2.349 & 0.001651 & 20.89 & 0.0079 \\
\hline-105.935 & 1.956 & 2.324 & 0.001650 & 83.74 & 0.067 \\
\hline-103.435 & 1.925 & 2.292 & 0.001649 & 24.60 & 0.0014 \\
\hline-102.935 & 1.918 & 2.285 & 0.001649 & 25.15 & 0.0056 \\
\hline-102.018 & 1.907 & 2.273 & 0.001648 & 27.57 & 0.0017 \\
\hline-100.268 & 1.885 & 2.249 & 0.001648 & 24.11 & 0.015 \\
\hline-99.116 & 1.871 & 2.233 & 0.001647 & 27.12 & 0.0014 \\
\hline-98.643 & 1.865 & 2.226 & 0.001647 & 30.56 & 0.0050 \\
\hline-96.742 & 1.842 & 2.198 & 0.001646 & 25.58 & 0.0013 \\
\hline-95.643 & 1.828 & 2.182 & 0.001646 & 32.32 & 0.0053 \\
\hline-93.496 & 1.801 & 2.149 & 0.001645 & 27.68 & 0.0058 \\
\hline-91.826 & 1.781 & 2.123 & 0.001644 & 27.02 & 0.0012 \\
\hline-89.452 & 1.751 & 2.085 & 0.001643 & 32.16 & 0.0011 \\
\hline-88.351 & 1.738 & 2.067 & 0.001642 & 27.45 & 0.0053 \\
\hline-86.204 & 1.711 & 2.030 & 0.001642 & 29.40 & 0.0054 \\
\hline-84.058 & 1.685 & 1.993 & 0.001641 & 29.62 & 0.0052 \\
\hline-81.058 & 1.648 & 1.938 & 0.001639 & 32.73 & 0.0050 \\
\hline-80.147 & 1.637 & 1.921 & 0.001639 & 34.20 & 0.00090 \\
\hline-78.911 & 1.622 & 1.898 & 0.001638 & 32.57 & 0.0047 \\
\hline-77.243 & 1.602 & 1.866 & 0.001638 & 39.66 & 0.00079 \\
\hline-76.765 & 1.596 & 1.857 & 0.001637 & 31.73 & 0.0047 \\
\hline-74.868 & 1.573 & 1.819 & 0.001620 & 37.35 & 0.00089 \\
\hline-73.765 & 1.560 & 1.797 & 0.001619 & 35.53 & 0.0046 \\
\hline-71.954 & 1.538 & 1.760 & 0.001618 & 38.91 & 0.0047 \\
\hline-69.808 & 1.512 & 1.716 & 0.001617 & 40.07 & 0.0042 \\
\hline-66.808 & 1.476 & 1.651 & 0.001616 & 43.95 & 0.0033 \\
\hline-64.995 & 1.455 & 1.611 & 0.001615 & 37.96 & 0.0034 \\
\hline
\end{tabular}

Table 2

(Continued)

\begin{tabular}{|c|c|c|c|c|c|}
\hline$\overline{\Delta T \text { (days) }}$ & $r(\mathrm{AU})$ & $\Delta(\mathrm{AU})$ & $g\left(\mathrm{~s}^{-1}\right)$ & $Q\left(10^{28} \mathrm{~s}^{-1}\right)$ & $\delta Q\left(10^{28} \mathrm{~s}^{-1}\right)$ \\
\hline-60.335 & 1.401 & 1.505 & 0.001599 & 45.97 & 0.0059 \\
\hline-59.827 & 1.395 & 1.493 & 0.001599 & 38.34 & 0.0033 \\
\hline-58.014 & 1.374 & 1.451 & 0.001598 & 37.54 & 0.0033 \\
\hline-55.868 & 1.350 & 1.399 & 0.001597 & 35.70 & 0.0036 \\
\hline-52.869 & 1.317 & 1.326 & 0.001596 & 28.57 & 0.0057 \\
\hline-51.056 & 1.297 & 1.281 & 0.001581 & 26.87 & 0.0037 \\
\hline-39.149 & 1.175 & 0.970 & 0.001549 & 30.09 & 0.0044 \\
\hline-37.797 & 1.162 & 0.933 & 0.001548 & 32.97 & 0.00077 \\
\hline-36.246 & 1.148 & 0.891 & 0.001534 & 29.67 & 0.0041 \\
\hline-34.895 & 1.135 & 0.855 & 0.001534 & 34.62 & 0.00069 \\
\hline-34.090 & 1.128 & 0.833 & 0.001534 & 31.93 & 0.0036 \\
\hline-32.520 & 1.115 & 0.790 & 0.001521 & 31.69 & 0.00069 \\
\hline-31.090 & 1.103 & 0.752 & 0.001521 & 31.26 & 0.0029 \\
\hline-30.506 & 1.098 & 0.736 & 0.001520 & 33.27 & 0.00062 \\
\hline-28.954 & 1.085 & 0.694 & 0.001508 & 29.10 & 0.0029 \\
\hline-26.809 & 1.069 & 0.637 & 0.001496 & 27.71 & 0.0028 \\
\hline-25.357 & 1.058 & 0.599 & 0.001496 & 54.85 & 0.0014 \\
\hline-17.220 & 1.008 & 0.409 & 0.001460 & 28.47 & 0.0014 \\
\hline-16.121 & 1.002 & 0.388 & 0.001460 & 29.61 & 0.0016 \\
\hline-13.982 & 0.993 & 0.355 & 0.001452 & 40.08 & 0.00089 \\
\hline-13.634 & 0.991 & 0.351 & 0.001452 & 28.82 & 0.0018 \\
\hline-11.607 & 0.983 & 0.331 & 0.001445 & 31.31 & 0.00055 \\
\hline-7.494 & 0.971 & 0.328 & 0.001432 & 37.63 & 0.0026 \\
\hline-5.109 & 0.966 & 0.350 & 0.001423 & 36.84 & 0.0027 \\
\hline-1.163 & 0.962 & 0.414 & 0.001416 & 30.22 & 0.0021 \\
\hline 1.476 & 0.962 & 0.471 & 0.001416 & 25.30 & 0.0026 \\
\hline 3.288 & 0.964 & 0.513 & 0.001413 & 20.95 & 0.0033 \\
\hline 5.290 & 0.966 & 0.563 & 0.001411 & 22.35 & 0.0032 \\
\hline 8.315 & 0.973 & 0.640 & 0.001409 & 22.10 & 0.0038 \\
\hline 10.139 & 0.978 & 0.688 & 0.001409 & 22.28 & 0.0040 \\
\hline 13.403 & 0.990 & 0.775 & 0.001411 & 18.73 & 0.0048 \\
\hline 16.422 & 1.004 & 0.855 & 0.001413 & 18.64 & 0.0052 \\
\hline 18.235 & 1.013 & 0.903 & 0.001415 & 19.12 & 0.0054 \\
\hline 20.390 & 1.026 & 0.960 & 0.001420 & 19.64 & 0.0051 \\
\hline 23.409 & 1.045 & 1.038 & 0.001426 & 16.93 & 0.0065 \\
\hline 25.222 & 1.057 & 1.084 & 0.001432 & 16.40 & 0.0076 \\
\hline 27.377 & 1.073 & 1.138 & 0.001439 & 16.61 & 0.0079 \\
\hline 30.376 & 1.097 & 1.211 & 0.001447 & 15.88 & 0.0079 \\
\hline 32.210 & 1.112 & 1.254 & 0.001447 & 16.14 & 0.0079 \\
\hline 34.355 & 1.131 & 1.305 & 0.001455 & 14.38 & 0.0085 \\
\hline 38.465 & 1.168 & 1.397 & 0.001463 & 11.90 & 0.12 \\
\hline 44.663 & 1.229 & 1.528 & 0.001471 & 13.28 & 0.014 \\
\hline 46.323 & 1.246 & 1.562 & 0.001481 & 14.94 & 0.014 \\
\hline 48.323 & 1.267 & 1.601 & 0.001480 & 15.52 & 0.014 \\
\hline 51.315 & 1.300 & 1.657 & 0.001480 & 14.92 & 0.015 \\
\hline 52.364 & 1.311 & 1.677 & 0.001479 & 13.15 & 0.016 \\
\hline 53.420 & 1.323 & 1.696 & 0.001490 & 16.28 & 0.015 \\
\hline 54.475 & 1.334 & 1.714 & 0.001490 & 19.07 & 0.015 \\
\hline 56.843 & 1.361 & 1.755 & 0.001489 & 20.74 & 0.012 \\
\hline 58.593 & 1.381 & 1.785 & 0.001489 & 26.49 & 0.010 \\
\hline 61.544 & 1.415 & 1.832 & 0.001499 & 29.83 & 0.010 \\
\hline 63.911 & 1.442 & 1.869 & 0.001498 & 28.59 & 0.011 \\
\hline 65.654 & 1.463 & 1.894 & 0.001498 & 25.41 & 0.012 \\
\hline 66.575 & 1.474 & 1.908 & 0.001497 & 24.12 & 0.0023 \\
\hline 72.639 & 1.546 & 1.990 & 0.001496 & 10.76 & 0.020 \\
\hline 75.508 & 1.581 & 2.026 & 0.001495 & 10.62 & 0.021 \\
\hline 77.875 & 1.609 & 2.054 & 0.001507 & 10.69 & 0.021 \\
\hline 79.625 & 1.631 & 2.074 & 0.001507 & 9.974 & 0.023 \\
\hline 80.575 & 1.642 & 2.084 & 0.001507 & 11.98 & 0.0040 \\
\hline 82.494 & 1.666 & 2.105 & 0.001506 & 9.284 & 0.023 \\
\hline 84.862 & 1.695 & 2.129 & 0.001506 & 9.804 & 0.023 \\
\hline 86.606 & 1.716 & 2.146 & 0.001505 & 10.37 & 0.022 \\
\hline 89.557 & 1.753 & 2.173 & 0.001505 & 8.652 & 0.025 \\
\hline 91.932 & 1.782 & 2.194 & 0.001504 & 6.097 & 0.030 \\
\hline 93.676 & 1.804 & 2.208 & 0.001504 & 7.022 & 0.029 \\
\hline 96.460 & 1.838 & 2.230 & 0.001503 & 6.228 & 0.029 \\
\hline
\end{tabular}


Table 2

(Continued)

\begin{tabular}{lccccc}
\hline \hline$\Delta T($ days $)$ & $r(\mathrm{AU})$ & $\Delta(\mathrm{AU})$ & $g\left(\mathrm{~s}^{-1}\right)$ & $Q\left(10^{28} \mathrm{~s}^{-1}\right)$ & $\delta Q\left(10^{28} \mathrm{~s}^{-1}\right)$ \\
\hline 100.050 & 1.883 & 2.256 & 0.001502 & 7.318 & 0.026 \\
102.939 & 1.918 & 2.276 & 0.001502 & 7.179 & 0.030 \\
105.314 & 1.948 & 2.291 & 0.001501 & 8.051 & 0.028 \\
107.072 & 1.970 & 2.301 & 0.001501 & 7.483 & 0.027 \\
109.951 & 2.005 & 2.317 & 0.001501 & 4.486 & 0.047 \\
111.348 & 2.023 & 2.325 & 0.001500 & 3.959 & 0.057 \\
120.780 & 2.139 & 2.369 & 0.001499 & 6.427 & 0.034 \\
126.378 & 2.208 & 2.392 & 0.001497 & 7.562 & 0.029 \\
138.835 & 2.361 & 2.437 & 0.001495 & 5.311 & 0.023 \\
141.209 & 2.390 & 2.446 & 0.001495 & 4.109 & 0.031 \\
147.704 & 2.469 & 2.469 & 0.001482 & 4.827 & 0.024 \\
148.759 & 2.482 & 2.473 & 0.001482 & 3.344 & 0.034 \\
151.877 & 2.519 & 2.486 & 0.001481 & 5.492 & 0.026 \\
152.933 & 2.532 & 2.490 & 0.001481 & 3.500 & 0.035 \\
153.988 & 2.545 & 2.494 & 0.001481 & 3.685 & 0.034 \\
170.986 & 2.748 & 2.581 & 0.001478 & 4.170 & 0.025
\end{tabular}

Notes.

$\Delta T$ : time from perihelion 2004 May 15.97, in days.

$r$ : heliocentric distance (AU).

$\Delta$ : geocentric distance (AU).

$g$ : solar Ly $\alpha$-factor (photons $\mathrm{s}^{-1}$ ).

$Q$ : water production rate $\left(10^{28} \mathrm{~s}^{-1}\right)$.

$\delta Q: 1 \sigma$ uncertainty $\left(10^{28} \mathrm{~s}^{-1}\right)$.

\section{DISCUSSION}

Q4 is a dynamically new Oort Cloud comet (Biver et al. 2007). The pre-perihelion variation seen in Figure 2, unlike many of the SWAN comets, is not characterized by a uniform overall "straight line" slope as in the best-fit power law, but rather is steeper than the power law for $r>2 \mathrm{AU}$ and somewhat more shallow for $r<1.3 \mathrm{AU}$. This is somewhat reminiscent of the seasonal variation resulting from an active spot seen in comet 19P/Borrelly and characterized by Schleicher et al. (2003). During most of the pre-perihelion period, roughly from 2.2 to $1.5 \mathrm{AU}$ the production rate has a broad enhancement above the mean power-law distribution. There was then a very large production rate spike (outburst) about 30 days before perihelion. The general trend after perihelion follows the straight-line power-law slope (see Figure 2) closely, except for a large extended increase above the nominal trend from 50 to 70 days after perihelion. The fact that the largest production rate is before perihelion and that there is a rise in the production rate $\sim 60$ days after perihelion is also reflected in the visual light curve (Yoshida, 2008), which peaks about 2 weeks before perihelion and which has a definite shoulder in the post-perihelion leg.

T7 does not show any peculiar activity changes or outbursts beyond having larger production rates before perihelion than after. The visual light curve (Yoshida 2008), although overall larger before perihelion than after, is consistent with the water

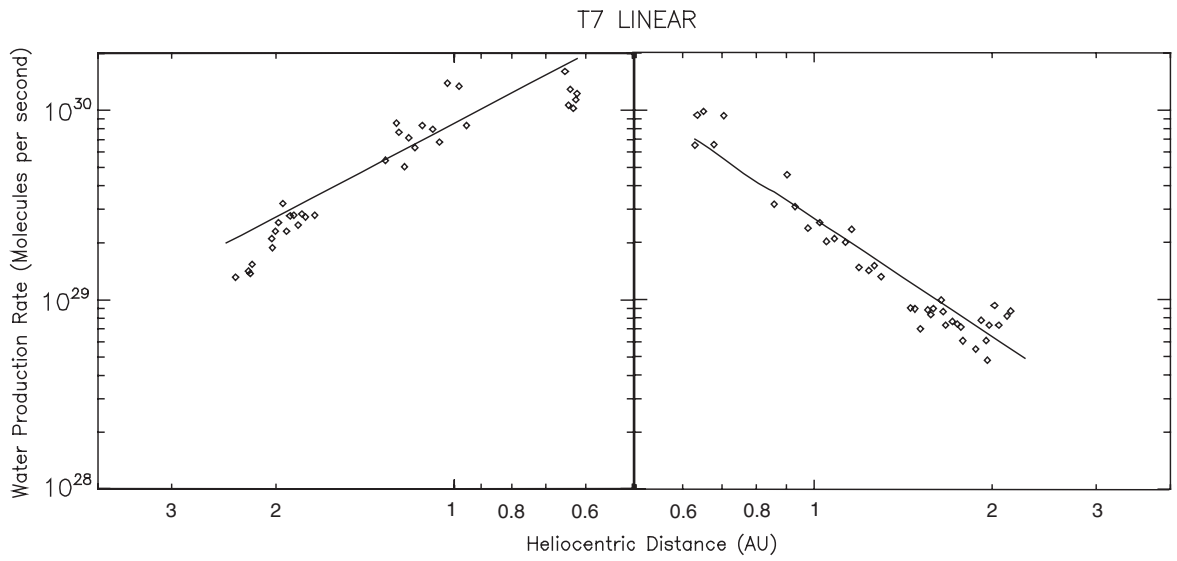

Figure 1. Single-image water production rate in C/2002 T7 (LINEAR) as a function of heliocentric distance. Shown as the points are the single-image water production rates determined from the SWAN images of the Ly $\alpha$ coma of comet C/2002 T7 (LINEAR). The straight lines give the best-fit power-law variations for the separate pre- (left) and post-perihelion (right) results, which are $8.4 \times 10^{29} r^{-1.6}$ and $2.7 \times 10^{29} r^{-2.1}$, respectively, in molecules s ${ }^{-1}$ with heliocentric distance, $r$, in AU.

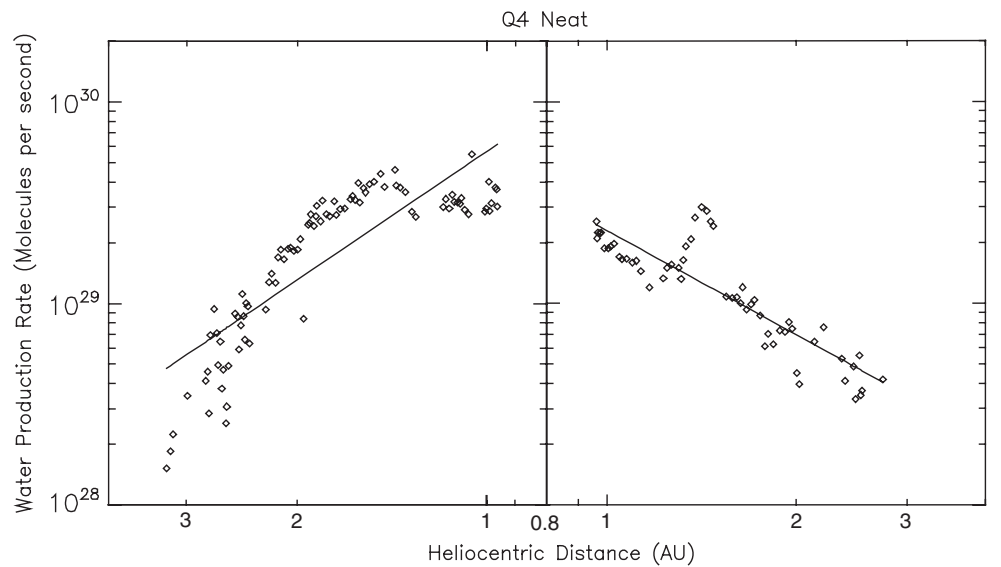

Figure 2. Single-image water production rate in C/2001 Q4 (NEAT) as a function of heliocentric distance. Shown as the points are the single-image water production rates determined from the SWAN images of the Ly $\alpha$ coma of comet C/2001 Q4 (NEAT). The straight lines give the best-fit power-law variations for the separate pre(left) and post-perihelion (right) results, which are $5.8 \times 10^{29} r^{-2.2}$ and $2.3 \times 10^{29} r^{-1.7}$, respectively, in molecules s ${ }^{-1}$ with heliocentric distance, $r$, in AU. 
Table 3

C/2002 T7 LINEAR: Deconvolved Daily-Average Water Production Rates

\begin{tabular}{lcc}
\hline \hline$\Delta T$ (days) & $Q\left(10^{28} \mathrm{~s}^{-1}\right)$ & $\delta Q\left(10^{28} \mathrm{~s}^{-1}\right)$ \\
\hline-134.076 & 14.65 & 13 \\
-133.076 & 14.28 & 13
\end{tabular}

$-132.076$

$-131.076$

$-130.076$

$-129.076$

$-128.076$

$-127.076$

$-126.076$

$-125.076$

$-124.076$

$-123.076$

$-122.076$

$-121.076$

$-120.076$

$-119.076$

$-118.076$

$-117.076$

$-116.076$

$-115.076$

$-114.076$

$-113.076$

$-112.076$

$-111.076$

$-110.076$

$-109.076$

$-108.076$

$-107.076$

$-106.076$

$-105.076$

$-104.076$

$-103.076$

$-102.076$

$-101.076$

$-100.076$

$-99.076$

$-98.076$

$-97.076$

$-96.076$

$-95.076$

$-94.076$

$-93.076$

$-92.076$

$-91.076$

$-84.076$

$-83.076$

$-82.076$

$-81.076$

$-80.076$

$-79.076$

$-78.076$

$-77.076$

$-76.076$

$-75.076$

$-74.076$

$-73.076$

$-72.076$

$-71.076$

$-70.076$

$-69.076$

$-68.076$

$-67.076$

$-66.076$

$-65.076$

$-64.076$

$-63.076$
15.19

16.76

18.27

16.68

18.78

16.81

17.37

17.67

19.17

16.82

17.25

17.41

18.18

17.70

18.26

18.46

17.49

15.94

17.47

19.60

19.49

20.20

20.22

22.50

23.37

24.26

25.73

26.36

27.92

27.05

28.29

26.56

27.14

27.87

28.81

25.74

27.53

27.67

27.41

27.90

27.83

27.59

29.49

29.95

27.97

27.83

27.51

27.20

26.99

26.73

26.48

26.41

26.24

26.10

39.33

39.57

40.25

18.93

21.22

23.76

26.46

29.49

32.70

38.64
11.

11.

9.1

9.3

6.8

7.6

5.0

7.9

4.8

8.3

4.9

15.

13.

12.

11.

11.

11.

11.

8.8

7.9

6.8

6.6

4.9

7.2

5.5

8.3

6.8

9.5

8.5

9.8

8.1

10.

7.8

9.1

6.0

9.8

12.

11.

8.4

13.

12.

10.

20.

20.

19.

19.

19.

19.

22.

22.

22.

17.

17.

17.

20.

19.

19.

22.

20.

18.

18.

15

14.

14.

Table 3

(Continued)

\begin{tabular}{|c|c|c|}
\hline$\Delta T$ (days) & $Q\left(10^{28} \mathrm{~s}^{-1}\right)$ & $\delta Q\left(10^{28} \mathrm{~s}^{-1}\right)$ \\
\hline-62.076 & 42.30 & 12. \\
\hline-61.076 & 45.48 & 12. \\
\hline-60.076 & 50.14 & 21. \\
\hline-59.076 & 51.26 & 18. \\
\hline-58.076 & 53.41 & 14. \\
\hline-57.076 & 40.87 & 15. \\
\hline-56.076 & 38.89 & 11. \\
\hline-55.076 & 34.71 & 4.3 \\
\hline-54.076 & 50.23 & 22. \\
\hline-53.076 & 49.56 & 18. \\
\hline-52.076 & 48.73 & 13. \\
\hline-51.076 & 54.19 & 22. \\
\hline-50.076 & 54.24 & 17. \\
\hline-49.076 & 55.18 & 10. \\
\hline-48.076 & 56.76 & 28. \\
\hline-47.076 & 56.96 & 23. \\
\hline-46.076 & 56.51 & 19. \\
\hline-13.076 & 175.2 & 140 \\
\hline-12.076 & 129.8 & 66. \\
\hline-11.076 & 107.5 & 24. \\
\hline-10.076 & 96.99 & 10. \\
\hline-9.076 & 104.1 & 1.4 \\
\hline-8.076 & 94.83 & 1.4 \\
\hline-4.076 & 82.60 & 24. \\
\hline-3.076 & 88.77 & 32. \\
\hline-2.076 & 71.10 & 19. \\
\hline-1.076 & 58.58 & 11. \\
\hline-0.076 & 59.23 & 7.5 \\
\hline 0.924 & 56.11 & 3.8 \\
\hline 1.924 & 49.38 & 2.1 \\
\hline 2.924 & 47.40 & 0.56 \\
\hline 3.924 & 54.86 & 0.31 \\
\hline 4.924 & 90.89 & 1.9 \\
\hline 5.924 & 71.60 & 1.9 \\
\hline 6.924 & 85.70 & 1.0 \\
\hline 7.924 & 116.3 & 1.5 \\
\hline 8.924 & 53.32 & 3.1 \\
\hline 9.924 & 52.28 & 1.3 \\
\hline 10.924 & 58.67 & 0.96 \\
\hline 11.924 & 59.30 & 1.4 \\
\hline 12.924 & 60.04 & 0.51 \\
\hline 13.924 & 64.08 & 0.55 \\
\hline 29.924 & 26.47 & 6.7 \\
\hline 30.924 & 27.11 & 6.8 \\
\hline 31.924 & 26.78 & 5.3 \\
\hline 32.924 & 25.45 & 2.1 \\
\hline 33.924 & 25.08 & 1.2 \\
\hline 34.924 & 27.14 & 3.1 \\
\hline 35.924 & 27.66 & 2.0 \\
\hline 36.924 & 30.64 & 2.2 \\
\hline 37.924 & 36.92 & 1.3 \\
\hline 38.924 & 22.43 & 0.68 \\
\hline 54.924 & 14.72 & 7.3 \\
\hline 55.924 & 14.22 & 7.0 \\
\hline 56.924 & 5.144 & 6.5 \\
\hline 57.924 & 5.540 & 6.3 \\
\hline 58.924 & 5.922 & 5.8 \\
\hline 59.924 & 6.391 & 5.4 \\
\hline 60.924 & 6.819 & 4.9 \\
\hline 61.924 & 7.441 & 4.6 \\
\hline 62.924 & 8.096 & 5.6 \\
\hline 63.924 & 8.973 & 4.7 \\
\hline 64.924 & 12.61 & 7.1 \\
\hline 65.924 & 11.65 & 5.5 \\
\hline 66.924 & 9.834 & 5.3 \\
\hline 67.924 & 8.340 & 2.8 \\
\hline
\end{tabular}


Table 3

(Continued)

\begin{tabular}{ccc}
\hline \hline$\Delta T($ days $)$ & $Q\left(10^{28} \mathrm{~s}^{-1}\right)$ & $\delta Q\left(10^{28} \mathrm{~s}^{-1}\right)$ \\
\hline 68.924 & 9.281 & 6.8 \\
69.924 & 9.404 & 6.6 \\
70.924 & 9.840 & 5.8 \\
73.924 & 9.731 & 9.7 \\
74.924 & 9.905 & 8.6 \\
75.924 & 10.26 & 8.5 \\
76.924 & 11.59 & 7.4 \\
77.924 & 10.64 & 7.5 \\
78.924 & 11.22 & 7.5 \\
79.924 & 9.691 & 8.9 \\
80.924 & 8.535 & 8.5 \\
81.924 & 8.882 & 7.9 \\
82.924 & 9.000 & 7.0 \\
83.924 & 9.371 & 6.9 \\
84.924 & 8.577 & 5.4 \\
85.924 & 8.148 & 4.0 \\
91.924 & 8.216 & 7.4 \\
93.924 & 8.942 & 8.2 \\
94.924 & 8.801 & 8.0 \\
95.924 & 8.330 & 6.7 \\
96.924 & 7.965 & 5.7 \\
97.924 & 7.421 & 4.0 \\
\hline
\end{tabular}

Notes.

$\Delta T$ : time from perihelion 2004 April 23.06, in days

$Q$ : water production rate $\left(10^{28} \mathrm{~s}^{-1}\right)$

$\delta Q: 1 \sigma$ uncertainty $\left(10^{28} \mathrm{~s}^{-1}\right)$

production and has its largest peak or a dust outburst about 30 days after perihelion. Such behavior is not seen in either the record of the single-image or the daily deconvolved water production rates.

As mentioned in the previous section, both comets were a factor of 2-3 more productive before perihelion than after, although both showed generally similar heliocentric distance dependencies before and after perihelion. Because of the size of the SWAN instrument field of view, we are limited to extracting nothing shorter than daily-average values of the production rate. Given typical rotation rates of comets then, we expect that the values are also rotationally averaged values. In the absence of any other complementary results at this point, the overall asymmetric production about perihelion likely points to a seasonal effect owing to the orientation of the spin axis as it varies with respect to the sun. The asymmetry could be due to either elongated nucleus shape or to the distribution of active regions on the surface.

There are a number of ongoing studies and surveys that examine the ratios of various comet gas species in order to classify comets into groups, and perhaps give clues to their location of origin in the solar system, or about their evolutionary history. The location of $\mathrm{SOHO}$ at the L1 point enables SWAN to provide useful measures of the activity on short day-to-day and long timescales, providing a true measure of the activity with orbital position, as well as providing a temporal context to most observing programs which have only limited and sporadic time coverage. Therefore, in addition to providing many water production rate values, we have been examining the variations of production rate over the whole orbit and classifying them according to the heliocentric distance power law. We have been comparing the absolute water production rate levels and
Table 4

C/2001 Q4 NEAT: Deconvolved Daily-Average Water Production Rates

\begin{tabular}{|c|c|c|}
\hline$\Delta T$ (days) & $Q\left(10^{28} \mathrm{~s}^{-1}\right)$ & $\delta Q\left(10^{28} \mathrm{~s}^{-1}\right)$ \\
\hline-212.029 & 1.818 & 1.7 \\
\hline-211.029 & 2.056 & 1.7 \\
\hline-180.029 & 5.091 & 3.9 \\
\hline-179.029 & 6.522 & 5.5 \\
\hline-178.029 & 6.090 & 4.8 \\
\hline-177.029 & 6.235 & 3.0 \\
\hline-172.029 & 9.609 & 7.2 \\
\hline-171.029 & 12.84 & 5.8 \\
\hline-168.029 & 8.064 & 2.9 \\
\hline-165.029 & 6.352 & 2.6 \\
\hline-163.029 & 5.712 & 6.7 \\
\hline-162.029 & 5.679 & 4.9 \\
\hline-153.029 & 8.487 & 8.1 \\
\hline-151.029 & 9.970 & 7.3 \\
\hline-150.029 & 12.12 & 3.4 \\
\hline-147.029 & 11.25 & 7.7 \\
\hline-129.029 & 15.10 & 10. \\
\hline-126.029 & 15.33 & 12. \\
\hline-125.029 & 15.71 & 12. \\
\hline-124.029 & 16.71 & 10. \\
\hline-123.029 & 18.11 & 8.4 \\
\hline-122.029 & 16.76 & 11. \\
\hline-121.029 & 17.30 & 10. \\
\hline-120.029 & 17.84 & 9.3 \\
\hline-119.029 & 18.08 & 10. \\
\hline-118.029 & 18.68 & 9.1 \\
\hline-117.029 & 19.44 & 7.3 \\
\hline-116.029 & 19.00 & 1.1 \\
\hline-115.029 & 19.18 & 10. \\
\hline-114.029 & 19.52 & 9.0 \\
\hline-113.029 & 20.15 & 9.6 \\
\hline-112.029 & 19.65 & 10. \\
\hline-111.029 & 20.41 & 8.5 \\
\hline-110.029 & 20.47 & 14. \\
\hline-109.029 & 20.91 & 13. \\
\hline-108.029 & 21.76 & 11. \\
\hline-107.029 & 20.16 & 18. \\
\hline-106.029 & 21.42 & 15. \\
\hline-105.029 & 23.36 & 11. \\
\hline-104.029 & 21.89 & 11. \\
\hline-103.029 & 24.86 & 9.1 \\
\hline-102.029 & 29.09 & 6.9 \\
\hline-101.029 & 23.42 & 11. \\
\hline-100.029 & 24.78 & 9.3 \\
\hline-99.029 & 26.42 & 7.4 \\
\hline-98.029 & 24.39 & 8.4 \\
\hline-97.029 & 24.32 & 7.2 \\
\hline-96.029 & 24.26 & 5.9 \\
\hline-95.029 & 26.15 & 9.1 \\
\hline-94.029 & 26.40 & 7.5 \\
\hline-93.029 & 26.92 & 5.2 \\
\hline-92.029 & 26.91 & 9.6 \\
\hline-91.029 & 26.78 & 8.1 \\
\hline-90.029 & 26.81 & 6.3 \\
\hline-89.029 & 26.95 & 7.9 \\
\hline-88.029 & 27.86 & 9.3 \\
\hline-87.029 & 28.10 & 5.9 \\
\hline-86.029 & 28.55 & 7.1 \\
\hline-85.029 & 28.61 & 5.6 \\
\hline-84.029 & 28.30 & 3.7 \\
\hline-83.029 & 27.37 & 6.3 \\
\hline-82.029 & 27.24 & 5.4 \\
\hline-81.029 & 27.29 & 3.8 \\
\hline-80.029 & 31.40 & 7.9 \\
\hline-79.029 & 31.11 & 5.9 \\
\hline 78.029 & 30.67 & 46 \\
\hline
\end{tabular}


Table 4

(Continued)

\begin{tabular}{|c|c|c|}
\hline$\Delta T$ (days) & $Q\left(10^{28} \mathrm{~s}^{-1}\right)$ & $\delta Q\left(10^{28} \mathrm{~s}^{-1}\right)$ \\
\hline-77.029 & 33.32 & 7.6 \\
\hline-76.029 & 35.28 & 8.4 \\
\hline-75.029 & 35.99 & 6.1 \\
\hline-74.029 & 34.49 & 10. \\
\hline-73.029 & 35.40 & 8.4 \\
\hline-72.029 & 27.36 & 6.3 \\
\hline-71.029 & 27.22 & 5.2 \\
\hline-70.029 & 25.13 & 4.0 \\
\hline-69.029 & 25.15 & 3.2 \\
\hline-68.029 & 24.70 & 2.9 \\
\hline-67.029 & 24.50 & 2.3 \\
\hline-66.029 & 29.51 & 5.2 \\
\hline-65.029 & 29.12 & 3.5 \\
\hline-64.029 & 37.52 & 6.7 \\
\hline-63.029 & 39.31 & 2.8 \\
\hline-62.029 & 31.89 & 8.9 \\
\hline-61.029 & 32.05 & 6.4 \\
\hline-60.029 & 29.01 & 7.0 \\
\hline-59.029 & 28.73 & 4.7 \\
\hline-58.029 & 25.07 & 8.4 \\
\hline-57.029 & 24.35 & 6.4 \\
\hline-56.029 & 25.89 & 5.9 \\
\hline-55.029 & 24.86 & 3.4 \\
\hline-54.029 & 28.33 & 13. \\
\hline-53.029 & 27.85 & 13. \\
\hline-52.029 & 27.15 & 12. \\
\hline-51.029 & 26.70 & 11. \\
\hline-50.029 & 25.74 & 9.7 \\
\hline-49.029 & 24.69 & 8.3 \\
\hline-48.029 & 24.86 & 6.9 \\
\hline-47.029 & 25.09 & 5.7 \\
\hline-46.029 & 24.42 & 4.2 \\
\hline-45.029 & 24.83 & 3.5 \\
\hline-44.029 & 23.88 & 2.7 \\
\hline-43.029 & 23.15 & 1.8 \\
\hline-42.029 & 26.74 & 16 \\
\hline-41.029 & 33.97 & 0.53 \\
\hline-40.029 & 24.74 & 0.94 \\
\hline-39.029 & 40.07 & 7.9 \\
\hline-38.029 & 56.31 & 6.4 \\
\hline-37.029 & 73.44 & 14. \\
\hline-36.029 & 68.40 & 7.1 \\
\hline-35.029 & 30.24 & 3.4 \\
\hline-33.029 & 38.29 & 1.3 \\
\hline-32.029 & 82.51 & 41. \\
\hline-31.029 & 30.52 & 1.8 \\
\hline-30.029 & 27.97 & 1.7 \\
\hline-29.029 & 31.17 & 0.99 \\
\hline-24.029 & 23.25 & 2.8 \\
\hline-23.029 & 22.76 & 1.7 \\
\hline-22.029 & 22.90 & 1.2 \\
\hline-21.029 & 23.77 & 0.68 \\
\hline-20.029 & 27.16 & 0.376 \\
\hline-19.029 & 38.36 & 0.22 \\
\hline-18.029 & 39.38 & 0.26 \\
\hline-17.029 & 33.18 & 0.97 \\
\hline-16.029 & 31.21 & 0.75 \\
\hline-14.029 & 23.94 & 0.10 \\
\hline-12.029 & 29.56 & 1.7 \\
\hline-11.029 & 28.89 & 0.44 \\
\hline-10.029 & 38.53 & 0.49 \\
\hline-9.029 & 48.42 & 0.64 \\
\hline-8.029 & 40.48 & 0.12 \\
\hline-7.029 & 41.86 & 0.64 \\
\hline-6.029 & 24.48 & 2.7 \\
\hline-5.029 & 24.45 & 1.2 \\
\hline
\end{tabular}

Table 4

(Continued)

\begin{tabular}{|c|c|c|}
\hline$\Delta T$ (days) & $Q\left(10^{28} \mathrm{~s}^{-1}\right)$ & $\delta Q\left(10^{28} \mathrm{~s}^{-1}\right)$ \\
\hline-4.029 & 29.28 & 0.57 \\
\hline-3.029 & 42.43 & 0.89 \\
\hline-2.029 & 22.32 & 1.4 \\
\hline-1.029 & 23.80 & 1.1 \\
\hline-0.029 & 30.19 & 0.75 \\
\hline 0.971 & 20.39 & 0.83 \\
\hline 1.971 & 20.78 & 0.56 \\
\hline 2.971 & 27.60 & 0.72 \\
\hline 3.971 & 21.41 & 1.9 \\
\hline 4.971 & 21.45 & 1.53 \\
\hline 5.971 & 21.55 & 0.78 \\
\hline 6.971 & 21.12 & 0.80 \\
\hline 7.971 & 24.29 & 1.0 \\
\hline 8.971 & 18.63 & 2.7 \\
\hline 9.971 & 18.50 & 1.5 \\
\hline 10.971 & 19.41 & 0.79 \\
\hline 11.971 & 18.14 & 2.1 \\
\hline 12.971 & 19.05 & 1.6 \\
\hline 13.971 & 20.95 & 1.2 \\
\hline 14.971 & 19.98 & 1.6 \\
\hline 15.971 & 21.51 & 1.1 \\
\hline 16.971 & 20.26 & 1.5 \\
\hline 17.971 & 22.56 & 1.4 \\
\hline 18.971 & 17.62 & 3.6 \\
\hline 19.971 & 18.62 & 7.0 \\
\hline 20.971 & 18.97 & 3.0 \\
\hline 21.971 & 18.26 & 7.0 \\
\hline 22.971 & 18.29 & 3.1 \\
\hline 23.971 & 18.59 & 8.7 \\
\hline 24.971 & 19.15 & 4.5 \\
\hline 25.971 & 17.47 & 10. \\
\hline 26.971 & 17.48 & 6.6 \\
\hline 27.971 & 17.16 & 13. \\
\hline 28.971 & 17.13 & 9.6 \\
\hline 29.971 & 16.75 & 13. \\
\hline 30.971 & 16.56 & 13. \\
\hline 39.971 & 16.19 & 1.5 \\
\hline 40.971 & 16.08 & 11. \\
\hline 41.971 & 17.08 & 14. \\
\hline 42.971 & 17.38 & 9.5 \\
\hline 43.971 & 17.02 & 12. \\
\hline 44.971 & 16.95 & 7.2 \\
\hline 45.971 & 18.41 & 13. \\
\hline 46.971 & 18.51 & 10. \\
\hline 47.971 & 18.87 & 10. \\
\hline 48.971 & 18.62 & 6.9 \\
\hline 49.971 & 21.23 & 6.2 \\
\hline 50.971 & 21.33 & 3.4 \\
\hline 51.971 & 23.31 & 10. \\
\hline 52.971 & 24.22 & 7.4 \\
\hline 53.971 & 26.16 & 11. \\
\hline 54.971 & 27.91 & 11. \\
\hline 55.971 & 23.92 & 16. \\
\hline 56.971 & 25.84 & 16. \\
\hline 57.971 & 23.61 & 14. \\
\hline 58.971 & 24.62 & 13. \\
\hline 59.971 & 26.70 & 13. \\
\hline 60.971 & 23.55 & 12. \\
\hline 61.971 & 24.23 & 9.8 \\
\hline 62.971 & 24.80 & 6.1 \\
\hline 64.971 & 21.49 & 18 \\
\hline 65.971 & 17.96 & 10. \\
\hline 66.971 & 15.91 & 7.6 \\
\hline 67.971 & 14.68 & 5.6 \\
\hline 68.971 & 14.18 & 3.9 \\
\hline 69.971 & 14.53 & 6.8 \\
\hline
\end{tabular}


Table 4

(Continued)

\begin{tabular}{|c|c|c|}
\hline$\Delta T$ (days) & $Q\left(10^{28} \mathrm{~s}^{-1}\right)$ & $\delta Q\left(10^{28} \mathrm{~s}^{-1}\right)$ \\
\hline 70.971 & 13.64 & 6.0 \\
\hline 71.971 & 13.35 & 4.5 \\
\hline 72.971 & 12.11 & 6.6 \\
\hline 73.971 & 12.00 & 4.5 \\
\hline 74.971 & 12.52 & 2.8 \\
\hline 75.971 & 11.21 & 7.2 \\
\hline 76.971 & 11.24 & 6.6 \\
\hline 77.971 & 11.36 & 5.6 \\
\hline 78.971 & 11.02 & 6.3 \\
\hline 79.971 & 11.30 & 5.6 \\
\hline 80.971 & 11.71 & 4.6 \\
\hline 81.971 & 10.32 & 6.4 \\
\hline 82.971 & 10.20 & 6.3 \\
\hline 83.971 & 10.20 & 5.9 \\
\hline 84.971 & 9.364 & 6.4 \\
\hline 85.971 & 9.148 & 6.2 \\
\hline 86.971 & 9.153 & 5.5 \\
\hline 87.971 & 8.909 & 5.8 \\
\hline 88.971 & 8.820 & 5.5 \\
\hline 89.971 & 9.006 & 4.6 \\
\hline 90.971 & 8.284 & 6.1 \\
\hline 91.971 & 8.263 & 5.6 \\
\hline 92.971 & 8.407 & 4.7 \\
\hline 93.971 & 7.799 & 6.8 \\
\hline 94.971 & 8.017 & 6.2 \\
\hline 95.971 & 8.466 & 5.3 \\
\hline 96.971 & 7.530 & 6.7 \\
\hline 97.971 & 7.890 & 6.0 \\
\hline 98.971 & 8.584 & 5.0 \\
\hline 99.971 & 7.936 & 5.7 \\
\hline 100.971 & 8.202 & 5.2 \\
\hline 101.971 & 9.007 & 4.4 \\
\hline 102.971 & 6.718 & 6.4 \\
\hline 103.971 & 7.148 & 5.9 \\
\hline 104.971 & 6.899 & 5.3 \\
\hline 105.971 & 6.313 & 6.0 \\
\hline 106.971 & 6.103 & 5.4 \\
\hline 107.971 & 6.156 & 4.1 \\
\hline 114.971 & 5.585 & 5.5 \\
\hline 115.971 & 6.111 & 5.0 \\
\hline 116.971 & 6.744 & 3.9 \\
\hline 117.971 & 5.785 & 4.2 \\
\hline 118.971 & 5.966 & 4.6 \\
\hline 119.971 & 6.632 & 4.1 \\
\hline 120.971 & 6.953 & 4.2 \\
\hline 121.971 & 7.466 & 4.0 \\
\hline 122.971 & 8.485 & 3.7 \\
\hline 123.971 & 7.114 & 4.2 \\
\hline 124.971 & 7.317 & 4.2 \\
\hline 125.971 & 7.523 & 4.2 \\
\hline 126.971 & 7.712 & 4.1 \\
\hline 127.971 & 7.922 & 4.2 \\
\hline 128.971 & 8.135 & 4.3 \\
\hline 129.971 & 6.904 & 3.7 \\
\hline 130.971 & 6.744 & 3.6 \\
\hline 131.971 & 6.600 & 3.6 \\
\hline 132.971 & 6.476 & 3.3 \\
\hline 133.971 & 6.177 & 3.6 \\
\hline 134.971 & 7.938 & 3.4 \\
\hline 135.971 & 7.162 & 3.2 \\
\hline 136.971 & 6.636 & 2.8 \\
\hline 137.971 & 6.376 & 2.3 \\
\hline 138.971 & 5.445 & 3.1 \\
\hline 139.971 & 5.022 & 3.1 \\
\hline 140.971 & 5.169 & 3.5 \\
\hline 141.971 & 5.158 & 3.5 \\
\hline
\end{tabular}

Table 4

(Continued)

\begin{tabular}{lcc}
\hline \hline$\Delta T$ (days) & $Q\left(10^{28} \mathrm{~s}^{-1}\right)$ & $\delta Q\left(10^{28} \mathrm{~s}^{-1}\right)$ \\
\hline 142.971 & 5.374 & 3.2 \\
143.971 & 5.737 & 2.7 \\
144.971 & 4.876 & 3.8 \\
145.971 & 4.840 & 3.7 \\
146.971 & 5.205 & 3.5 \\
147.971 & 4.420 & 3.7 \\
148.971 & 4.648 & 3.5 \\
149.971 & 4.978 & 3.0 \\
\hline
\end{tabular}

Notes.

$\Delta T$ : time from perihelion 2004 May 15.97, in days. $Q$ : water production rate $\left(10^{28} \mathrm{~s}^{-1}\right)$. $\delta Q: 1 \sigma$ uncertainty $\left(10^{28} \mathrm{~s}^{-1}\right)$.

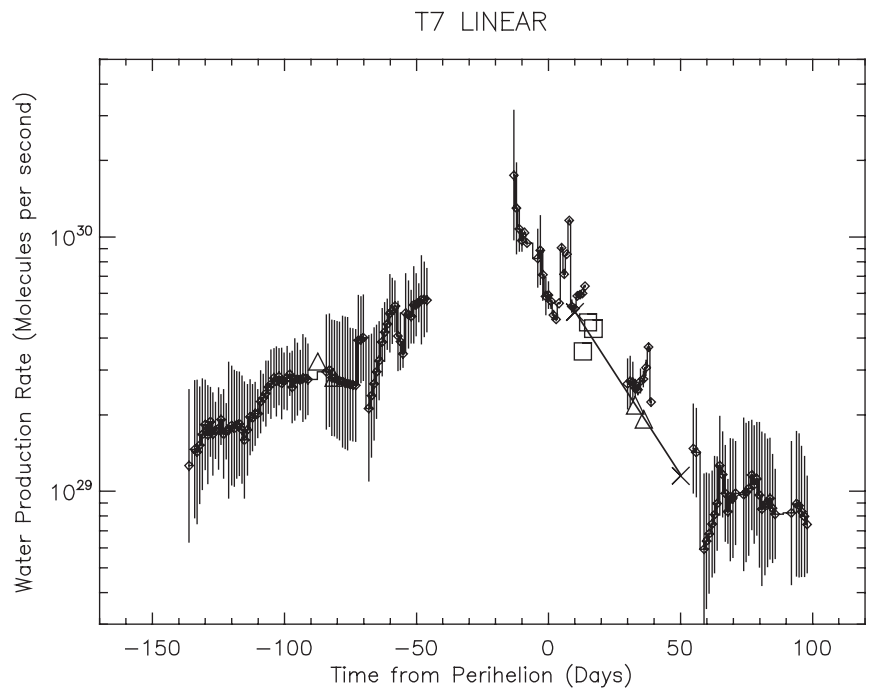

Figure 3. TRM deconvolved daily-average water production rate of $\mathrm{C} / 2002$ T7 (LINEAR) as a function of time. Shown as the diamonds connected by a histogram line are the daily-average water production rates of comet C/2002 T7 (LINEAR) determined with the time-resolved model from the SWAN images. The vertical lines correspond to the formal fitted model error bars. The large triangles are the Odin water production from Biver et al. (2007), the X's and line are from Anderson et al. (2008), and the squares are from DiSanti et al. (2006).

power-law slopes with various volatile composition measures elsewhere to look for any trends or correlations.

Five Oort Cloud comets, 1999 H1 Lee, 1999 T1 McNaughtHartley, 2000 WM1 LINEAR, 2001 A2 LINEAR, and 2002 C1 Ikeya-Zhang (P153) were also observed with SWAN (Combi et al. 2008) and analyzed in the same way as Q4 and T7. These were compared with previous SWAN observations of 1996 B2 Hyakutake (Combi et al. 2005), 1999 S4 LINEAR (Mäkinen et al. 2001), 2006 P1 McNaught (Neugebauer et al. 2007), and Hale-Bopp (Combi et al. 2000, 2006). It was found that the comets fell into three groups according to their power-law slopes: (1) shallow with $p \sim-1.5$, (2) nominal $(-3<p<-2)$, and (3) steep $(p<-3.5)$. This classification scheme would put $\mathrm{T} 7$ in the shallow group and Q4 in the nominal group. Table 5 gives an updated version of our summary of water production rates and power-law slopes for the SWAN comets published to date including Q4 and T7. In most of these comets, there were pre- to post-perihelion asymmetries in overall production level and some irregular variations owing to outbursts, e.g., for $\mathrm{C} /$ 2000 A2 LINEAR (Combi et al. 2008), and seasonal effects from either the nucleus shape or the distribution of active areas, e.g., 


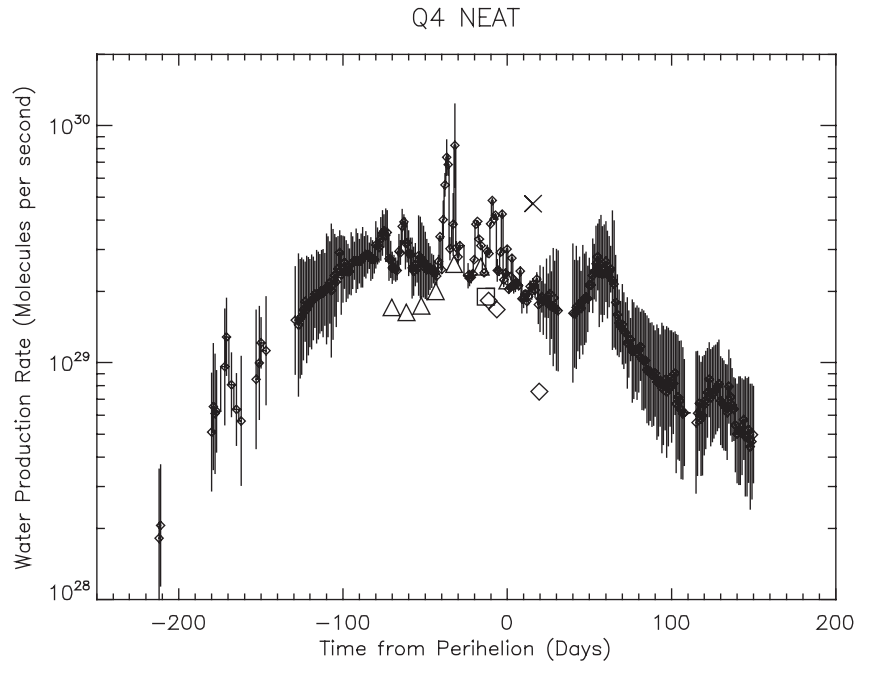

Figure 4. TRM deconvolved daily-average water production rate of C/2001 Q4 (NEAT) as a function of time. Shown as the diamonds connected by a histogram line are the daily-average water production rates of comet C/2001 Q4 (NEAT) determined with the time-resolved model from the SWAN images. The vertical lines correspond to the formal fitted model error bars. The large triangles are the Odin water production from Biver et al. (2007), the large diamonds are from J. Crovisier et al. (2009, in preparation), the $\mathrm{X}$ is from Anderson et al. (2008), and the large square is from Weaver et al. (2004).

for Hyakutake (Combi et al. 2005). However, with the advantage of having a consistent set of water production rate over a large portion of the apparition, our results are able to place peculiar variations (short and intermediate term) into proper context and reveal that the slopes of the underlying variations were generally similar for the same comet over the whole apparition.

T7 and Q4 were two moderately bright comets observed during the same general time period; a number of publications, like this one, have included observations of both and compar- isons of them together and with other comets. Remijan et al. (2006), based on observations of several molecules including $\mathrm{HCN}$ and $\mathrm{CH}_{3} \mathrm{OH}$, concluded that $\mathrm{T} 7$ is compositionally similar to Hale-Bopp, while Q4 is more similar to Hyakutake, with T7 and Hale-Bopp being richer in $\mathrm{HCN}$ and $\mathrm{CH}_{3} \mathrm{OH}$ compared to water and Q4 and Hyakutake being poorer in both. M. A. DiSanti et al. (2008, private communication) find that Q4 has abundances of $\mathrm{CO}$ and $\mathrm{C}_{2} \mathrm{H}_{6}$ relative to water of $3 \%-$ $9 \%$ and $\sim 0.5 \%$, respectively, and that $\mathrm{T} 7$ has abundances of these species of $2 \%-3 \%$ and $\sim 0.5 \%$, respectively. In contrast, both Hale-Bopp and Hyakutake were much more enriched in CO, 23\% and 19\%-30\%, respectively, compared with either Q4 or T7. Milam et al. (2006) find both T7 and Q4 depleted in formaldehyde, compared with Hale-Bopp, but similar to 1P/ Halley. Lupu et al. (2007) find a CO production in Q4 relative to water of $8.8 \% \pm 0.8 \%$ from $H S T$ observations. Finally, Gibb et al. (2007) find that $\mathrm{CO}$ and $\mathrm{CH}_{4}$ do not correlate in a sample of eight comets including Q4 and T7. Table 5 gives an updated version of our summary of water production rate power-law slopes for the SWAN comets published to date compared with the abundances of $\mathrm{CO}$ and $\mathrm{C}_{2} \mathrm{H}_{6}$ compared with water.

\section{SUMMARY}

We present single-image values and sequences of dailyaverage values of the water production rate in comets $C / 2001$ Q4 NEAT and C/2002 T7 LINEAR obtained by analysis of $S O H O$ SWAN images of their H Ly $\alpha$ comae. The average powerlaw fit to the production rate variation of Q4 gives $3.5 \times 10^{29}$ $r^{-1.7}$ and that for $\mathrm{T} 7$ gives $4.6 \times 10^{29} r^{-2.0}$. Q4 showed a very large production rate spike about 30 days before perihelion and then a large extended increase above the nominal trend from 50 to 70 days after perihelion. T7 showed no large outbursts. Both comets were more productive at comparable heliocentric distances before perihelion than after. In terms of the production

Table 5

Volatile Composition for Three Water Variation Groups in SWAN Comets

\begin{tabular}{|c|c|c|c|c|}
\hline Comet & $Q_{1}\left(\mathrm{H}_{2} \mathrm{O}\right)^{\mathrm{a}}$ & $p^{\mathrm{b}}$ & $\mathrm{CO} / \mathrm{H}_{2} \mathrm{O}$ & $\mathrm{C}_{2} \mathrm{H}_{6} / \mathrm{H}_{2} \mathrm{O}$ \\
\hline \multicolumn{5}{|c|}{ Steep slope } \\
\hline C 1999 T1 McNaught-Hartleyc & $2.9 \times 10^{29}$ & -3.3 & $15 \%(1)$ & $0.6 \%(2)$ \\
\hline C 2001 A2 LINEAR ${ }^{c}$ & $8.2 \times 10^{28}$ & -4.5 & $1.5 \%(1,3)$ & $1.6 \%(3)$ \\
\hline \multicolumn{5}{|c|}{ Moderate slope } \\
\hline C 1995 OI Hale-Bopp ${ }^{\mathrm{d}}$ & $1.3 \times 10^{31}$ & -2.6 & $23 \%(4,5)$ & $0.6 \%(2)$ \\
\hline C 1999 H1 Lee & $1.5 \times 10^{29}$ & -2.7 & $2-4 \%(6,7)$ & $0.7 \%(8)$ \\
\hline P153/Ikeya-Zhang ${ }^{\mathrm{c}}$ & $1.8 \times 10^{29}$ & -2.7 & $4 \%(9,10)$ & $0.6 \%(10)$ \\
\hline C 1996 B2 Hyakutake ${ }^{\mathrm{e}}$ & $2.7 \times 10^{29}$ & -2.1 & $19-30 \%(11-14)$ & $0.6 \%(15)$ \\
\hline C 2002 T7 LINEAR ${ }^{f}$ & $4.6 \times 10^{29}$ & -2.0 & $2-3 \%$ & $\sim 0.5 \%$ \\
\hline \multicolumn{5}{|c|}{ Shallow slope } \\
\hline C 1999 S4 LINEAR ${ }^{g}$ & $1.7 \times 10^{28}$ & -1.6 & $0.6 \%(17,18)$ & $0.1 \%(18)$ \\
\hline C 2001 WM1 LINEAR ${ }^{c}$ & $1.1 \times 10^{29}$ & -1.4 & $1 \%(1,9)$ & $0.5 \%(19)$ \\
\hline C 2006 P1 McNaught ${ }^{\mathrm{h}}$ & $9.2 \times 10^{29}$ & -1.7 & $2-4 \%(20)$ & - \\
\hline C 2001 Q4 NEAT & $3.5 \times 10^{29}$ & -1.7 & $3-9 \%(16)$ & $\sim 0.5 \%$ \\
\hline
\end{tabular}

Notes.

Note that the new results for T7 and Q4 are shown in bold type.

${ }^{a}$ Average pre-post water production rate at $1 \mathrm{AU}$ in molecules s ${ }^{-1}$.

${ }^{\mathrm{b}}$ Average pre-post power-law exponent.

References for SWAN water production rates and slopes: ${ }^{\mathrm{c}}$ Combi et al. (2008); ${ }^{\mathrm{d}}$ Combi et al. (2000); ${ }^{\mathrm{e}}$ Combi et al. (2005); ${ }^{\mathrm{f}} \mathrm{This}$ paper; g Mäkinen et al. (2001); hNeugebauer et al. (2007).

References for volatile abundances: (1) Biver et al. 2006; (2) Dello Russo et al. 2001; (3) Magee-Sauer et al. 2008; (4) DiSanti et al. 2001; (5) Bockelée-Morvan et al. 2000; (6) Biver et al. 2000; (7) Mumma et al. 2001a; (8) Mumma et al. 2001b; (9) Weaver et al. 2002; (10) DiSanti et al. 2002; (11) DiSanti et al. 2003; (12) McPhate et al. 1996; (13) Biver et al. 1999; (14) Lis et al. 1997; (15) Mumma et al. 1996; (16) M. A. DiSanti et al. 2008 (private communication); (17) Weaver et al. 2001; (18) Mumma et al. 2001a; (19) Radeva et al. 2008; (20) Dello Russo et al. 2007. 
rate power-law slope classification by Combi et al. (2008), Q4 falls in the shallow slope group and $\mathrm{T} 7$ falls in the moderate slope group. So far our comparison of slopes and production rates level shows no obvious correlations with either $\mathrm{CO}$ or $\mathrm{C}_{2} \mathrm{H}_{6}$ abundance variations. In the future, we will expand our comparisons with other detected species as well as $\operatorname{Af} \rho$ and the standard visual radical composition groups.

$\mathrm{SOHO}$ is an international cooperative mission between ESA and NASA. M. Combi acknowledges support from grants NNG05GF06G and NNG08A044G from the NASA Planetary Astronomy Program. J.-L. Bertaux and E. Quémerais acknowledge support from CNRS and CNES. J.T.T. Mäkinen was supported by the Finnish Meteorological Institute. We obtained cometary ephemerides from the JPL Horizons Web site. We also acknowledge the personnel that have been keeping $\mathrm{SOHO}$ and SWAN operational for 13 years. We thank Nathaniel Henry who helped with the data reduction. We also thank the referee, Lori Feaga, who made a number of helpful comments and criticisms that have greatly improved the paper.

\section{REFERENCES}

Anderson, W. M., DiSanti, M. A., Mumma, M. J., Bonev, B. P., Villaneuva, G. L., Dello Russo, N., Magee-Sauer, K., \& Gibb, E. L. 2008, in LPI Contribution 1405, Asteroids, Comets, Meteors held 2008 July 14-18, Baltimore, MD, paper 8387

Bertaux, J.-L., et al. 1997, Solar Physics, 175, 737

Biver, N., et al. 1999, AJ, 118, 1850

Biver, N., et al. 2000, AJ, 120, 1554

Biver, N., et al. 2006, A\&A, 449, 1255

Biver, N., et al. 2007, Planet. Space Sci., 55, 1058

Bockelée-Morvan, D., et al. 2000, A\&A, 353, 1101

Combi, M. R., Mäkinen, J. T. T., Bertaux, J.-L., \& Quémerais, E. 2005, Icarus, 177,228

Combi, M. R., Mäkinen, J. T. T., Henry, N. J., Bertaux, J.-L., \& Quémerais, E. 2006, BAAS, 38, 535

Combi, M. R., Mäkinen, J. T. T., Henry, N. J., Bertaux, J.-L., \& Quémerais, E. 2008, AJ, 135, 1533

Combi, M. R., Reinard, A. A., Bertaux, J.-L., Quémerais, E., \& Mäkinen, T. 2000, Icarus, 144, 191
Combi, M. R., \& Smyth, W. H. 1988, ApJ, 327, 1044

Dello Russo, N., Mumma, M. J., DiSanti, M. A., Magee-Sauer, K., \& Novak, R. 2001, Icarus, 153, 162

Dello Russo, N., Vervack, R. J. Jr., Weaver, H. A., \& Lisse, C. M. 2007, BAAS, 39,507

DiSanti, M. A., Bonev, B. P., Magee-Sauer, K., Dello Russo, N., Mumma, M. J., Reuter, D. C., \& Villanueva, G. L. 2006, ApJ, 650, 470

DiSanti, M. A., Dello Russo, N., Magee-Sauer, K., Gibb, E. L., Reuter, D. C., \& Mumma, M. J. 2002, in Proc. Asteroids Comets Meteors 2002, ed. B. Warmbein (ESA SP-5000; Noordwijk: ESA), 571

DiSanti, M. A., Mumma, M. J., Dello Russo, N., \& Magee-Sauer, K. 2001, Icarus, 153, 361

DiSanti, M. A., Mumma, M. J., Dello Russo, N., Magee-Sauer, K., \& Griep, D. 2003, J. Geophys. Res., 108, 5061

Festou, M. C. 1981, A\&A, 95, 69

Gibb, E., Bonev, B. P., Mumma, M. J., DiSanti, M. A., Magee-Sauer, K., \& Villanueva, G. 2007, BAAS, 38, 522

Keller, H. U., \& Meier, R. R. 1976, A\&A, 52, 273

Lis, D. C., et al. 1997, Earth Moon Planets, 87, 13

Lupu, R. E., Feldman, P. D., Weaver, H. A., \& Tozzi, G.-P. 2007, ApJ, 670, 1473

Magee-Sauer, K., Mumma, M. J., DiSanti, M. A., Dello Russo, N., Gibb, E. L., \& Bonev, B. P. 2008, Icarus, 194, 347

Mäkinen, J. T. T., Bertaux, J.-L., Combi, M. R., \& Quémerais, E. 2001, Science, 292, 1326

Mäkinen, J. T. T., Bertaux, J.-L., Combi, M. R., \& Quémerais, E. 2007, Icarus, 187,109

Mäkinen, J. T. T., \& Combi, M. R. 2005, Icarus, 177, 217

McPhate, J. B., Feldman, P. D., Weaver, H. A., A'Hearn, M. F., Tozzi, G.-P., \& Festou, M. C. 1996, BAAS, 28, 1093

Milam, S. N., et al. 2006, ApJ, 649, 1169

Mumma, M. J., DiSanti, M. A., Dello Russo, N., Fomenkova, M., Magee-Sauer, K., Kaminski, C. D., \& Xie, D. X. 1996, Science, 272, 1310

Mumma, M. J., et al. 2001a, Science, 292, 1334

Mumma, M. J., et al. 2001b, ApJ, 546, 1183

Neugebauer, M., et al. 2007, ApJ, 667, 1262

Radeva, Y. L., Mumma, M. J., Bonev, B. P., DiSanti, M. A., Villanueva, G. L. B. P., Magee-Sauer, K., Gibb, E. L., \& Weaver, H. A. 2008, BAAS, 40, 416

Remijan, A. J., et al. 2006, ApJ, 643, 567

Schleicher, D. G., Woodney, L. M., \& Millis, R. L. 2003, Icarus, 162, 415

Weaver, H. A., A'Hearn, M. F., Arpigny, C., Combi, M. R., Feldman, P. D., Festou, M. C., \& Tozzi, G.-P. 2004, BAAS, 36, 1120

Weaver, H., Feldman, P. D., Combi, M. R., Krasnopolsky, V., Lisse, C. M., \& Shemansky, D. E. 2002, ApJ, 576, L95

Weaver, H. A., et al. 2001, Science, 292, 1329

Yoshida, S. 2008, MISAO Project Comet Catalog, http://www.aerith.net/ comet/catalog/index-T-earth.html 